\title{
Comparison of international guidelines for noninvasive diagnosis of hepatocellular carcinoma: 2018 update
}

\author{
Tae-Hyung Kim ${ }^{1,2}$, So Yeon $\mathrm{Kim}^{3}$, An Tang ${ }^{4}$, and Jeong Min Lee ${ }^{1,2,5}$ \\ 'Department of Radiology, Seoul National University Hospital, Seoul; ${ }^{2}$ Department of Radiology, Seoul National University College of \\ Medicine, Seoul; ${ }^{3}$ Department of Radiology and Research Institute of Radiology, Asan Medical Center, University of Ulsan College of \\ Medicine, Seoul, Korea; ${ }^{4}$ Department of Radiology, Centre hospitalier de l'Université de Montréal (CHUM), Québec, Canada; ${ }^{5}$ Institute of \\ Radiation Medicine, Seoul National University Medical Research Center, Seoul, Korea
}

The goal of this review is to present the similarities and differences among the latest guidelines for noninvasive diagnosis of hepatocellular carcinoma (HCC) of American Association for the Study of Liver Disease (AASLD), European Association for the Study of the Liver (EASL), Liver Imaging Reporting and Data System (LI-RADS), Asian Pacific Association for the Study of the Liver (APASL), and Korean Liver Cancer Association-National Cancer Center (KLCA-NCC) of Korea. In 2018, major guideline updates have been proposed by the AASLD, EASL and KLCA-NCC; AASLD newly incorporated LI-RADS into their HCC diagnostic algorithm. The AASLD and EASL guidelines now include magnetic resonance imaging (MRI) using hepatobiliary contrast media as a first-line diagnostic test in addition to dynamic computed tomography and MRI using extracellular contrast media and the KLCA-NCC and EASL guidelines also include contrast-enhanced ultrasound as a second-line diagnostic test. We will comprehensively review the HCC surveillance and diagnostic algorithms and compare and highlight key features for each guideline. We also address limitations of current systems for the noninvasive diagnosis of HCC. (Clin Mol Hepatol 2019;25:245-263)

Keywords: Hepatocellular carcinoma; Diagnosis; Guideline; Standards

\section{INTRODUCTION}

Hepatocellular carcinoma (HCC) is the most common primary liver cancer in adults and the second leading cause of cancer-related death worldwide.' HCC occurs primarily in patients with chronic liver disease with various etiologies such as viral hepatitis, alcohol-induced liver disease, and nonalcoholic steatohepatitis. ${ }^{2}$ As curative treatment options for HCC including surgical resec- tion, liver transplantation, and ablation treatments are effective for early-stage HCC, timely diagnosis and early intervention are crucial in the management of patients with HCC. ${ }^{3}$ Considering that HCC can be diagnosed noninvasively using imaging tests without confirmatory biopsy ${ }^{4}$ and that imaging-based staging is essential for treatment selection, imaging plays critical role in management of HCC.

In order to standardize the acquisition, interpretation, and re-

\footnotetext{
Abbreviations:

AASLD, Association for the Study of Liver Diseases; AFP, alpha-fetoprotein; APASL, Asian-Pacific Association for the Study of the Liver; APHE, arterial phase hyperenhancement; BCLC, Barcelona Clinic Liver Cancer; CCs, cholangiocarcinomas; CEUS, contrast enhanced ultrasound; $C T$, computed tomography; EASL, European Association for the Study of the Liver; ECA, extracellular contrast agents; HBA, hepatobiliary contrast agents; HBP, hepatobiliary phase; HCC, hepatocellular carcinoma; KLCA-NCC, Korean Liver Cancer Association-National Cancer Center; LI-RADS, Liver Imaging Reporting and Data System; MRI, magnetic resonance imaging; mUICC, Modified Union for International Cancer Control; MRP2, multidrug resistanceassociated protein; OATP, organic-anion-transporting polypeptide; US, ultrasonography
}

\section{Corresponding author : Jeong Min Lee}

Department of Radiology, Seoul National University College of Medicine, 103 Daehak-ro, Jongno-gu, Seoul 03080, Korea

Tel: +82-2-2072-2254, Fax: +82-2-743-6385

E-mail:jmlshy2000@gmail.com

https://orcid.org/0000-0003-0561-8777 
porting of liver imaging examinations, several scientific organizations have proposed imaging-based diagnostic systems for surveillance, diagnosis, staging, treatment, and monitoring of treatment response of HCC. 5,6 The designs of these HCC imaging systems have varied between geographic areas so as to address different target populations, resources, and treatment practices. ${ }^{5}$ Despite these regional variations, HCC guidelines with imagingbased surveillance and diagnostic components have continued to improve the consistency and standardization of the acquisition, interpretation, and reporting of liver examinations. ${ }^{5,7}$ These guidelines have also been continually updated to reflect the most recent radiological and technological advances, as well as our better understanding of HCC pathophysiology. In 2017, the Asian Pacific Association for the Study of the Liver (APASL) practice guidelines have been updated. ${ }^{8}$ The Liver Imaging Reporting and Data System (LI-RADS) endorsed by the American College of Radiology, have been updated two years in a row, in $2017^{9}$ and 2018. In 2018, American Association for the Study of Liver Diseases (AASLD) — which released two distinct documents in the same year: HCC practice guidelines and practice guidance documents in the same year- ${ }^{4,11}$ integrated LI-RADS version 2018 into their HCC clinical practice guidance document; European Association for the Study of the Liver (EASL) ${ }^{12}$ updated their clinical practice guidelines for the management of HCC; and Korean Liver Cancer Association-National Cancer Center (KLCA-NCC) released their guidelines $v 2018$ in Korean ${ }^{13}$ and the English version is expected to be published soon.

The purpose of this review article is to synthesize and critically appraise the currently published imaging-based diagnostic systems endorsed by the five major societies (KLCA-NCC, APASL, AASLD, LI-RADS, EASL) for the noninvasive diagnosis of HCC and to address some limitations of current systems. Similar to the HCC diagnostic guidelines reviews by Cruite et al. in 2013, ${ }^{14}$ we converted the display from an algorithmic format of diagnostic systems to lists, while respecting and maintaining the original content in Tables 1 and 2.

\section{SURVEILLANCE}

Summaries of five HCC surveillance guidelines are provided in Table 1.

\section{Target population for surveillance}

All current guidelines recommend HCC surveillance in patients with liver cirrhosis or other risk factors for developing HCC. However, target populations differ in detail according to the guidelines. For example, KLCA-NCC include chronic HCV carrier whereas AASLD, EASL and APASL include cirrhotic patients with HCV. ${ }^{4,811,12}$ Ultrasound LI-RADS v $2017^{15}$ defers to regional HCC clinical practice guidelines for enrollment of adults with cirrhosis of any etiology and chronic hepatitis B or chronic hepatitis C viral infection even in the absence of cirrhosis.

\section{Tests for surveillance}

Ultrasound every 6 months is universally recommended for HCC surveillance in all guidelines. However, KLCA-NCC and APASL ${ }^{8}$ surveillance recommendations differ in that they utilize alpha-fetoprotein (AFP) as a tumor marker for surveillance in combination with the ultrasound, whereas use of AFP for surveillance is optional as per AASLD ${ }^{4}$ and LI-RADS guidelines ${ }^{15}$, and not recommended by EASL due to suboptimal cost-effectiveness for routine surveillance of early HCC. ${ }^{12}$ AFP and ultrasound have imperfect sensitivity, but may play complementary roles when combined. ${ }^{16}$ According to previous meta-analyses by Singal et al. ${ }^{17}$ and Tzartzeva et al. ${ }^{18}$, the sensitivity for detection of HCC using a combination of ultrasound and AFP is higher than that of ultrasound alone (respectively: $70 \%$ vs. $63 \%[P=0.65]^{17}, 63 \%$ vs. $45 \%[P=0.002]$ ). ${ }^{18}$ The differences in the surveillance test among guidelines may be due to the fact that the efficacy of the surveillance method can vary depending on the thresholds and the incidence of HCC in a given region. In North America and Europe, where the prevalence is relatively low, only ultrasound is recommended as a screening method, but in Asia, including Korea, where the prevalence of HCC is high, it is recommended to perform an ultrasound and AFP measurement combined.

Also, EASL further specifies ultrasound within 4 months when a nodule smaller than $1 \mathrm{~cm}$ has been detected, and EASL, AASLD, and KLCA-NCC recommend consideration of computed tomography (CT) or magnetic resonance imaging (MRI) for surveillance in select patients with a high likelihood of having an inadequate ultrasound or if ultrasound is attempted but inadequate (for example due to obesity, chest wall deformity, or intestinal gas). 
Table 1. Summary of surveillance for hepatocellular carcinoma in five different guidelines

\begin{tabular}{|c|c|c|c|c|c|}
\hline Organizations & KLCA-NCC $2018^{13}$ & APASL $2017^{8}$ & AASLD $2018^{11}$ & LI-RADS $2018^{10}$ & EASL $2018^{12}$ \\
\hline $\begin{array}{l}\text { Target } \\
\text { population for } \\
\text { surveillance }\end{array}$ & $\begin{array}{l}\text { - Cirrhotic patients } \\
\text { with varying } \\
\text { etiology } \\
\text { - Chronic HBV or } \\
\text { HCV carrier }\end{array}$ & $\begin{array}{l}\text { - Cirrhotic patients } \\
\text { with varying } \\
\text { etiology (HBV, HCV, } \\
\text { NASH, genetic } \\
\text { hemochromatosis, } \\
\text { PBC, alpha-1 } \\
\text { antitrypsin } \\
\text { deficiency) } \\
\text { • Non-cirrhotic HBV } \\
\text { patients (Asian men } \\
>40 \text { y, Asian women } \\
>50 \text { y, Africans }>20 \\
\text { y; family history of } \\
\text { HCC) }\end{array}$ & $\begin{array}{l}\text { - Cirrhotic patients } \\
\text { with varying etiology } \\
\text { (hepatitis B, hepatitis } \\
\text { C, primary biliary } \\
\text { cirrhosis, genetic } \\
\text { hemochromatosis, } \\
\text { alpha-1-antitrypsin) } \\
\text { - Hepatitis B carriers } \\
\text { (Asian men >40 y, Asian } \\
\text { women > } 50 \text { y, all } \\
\text { cirrhotic HBV carriers, } \\
\text { family history of HCC, } \\
\text { African/North } \\
\text { American blacks) }\end{array}$ & $\begin{array}{l}\text { - Cirrhotic patients with } \\
\text { any etiology } \\
\text { - Noncirrhotic HBV } \\
\text { carriers (Asian men } \\
>40 \text { y, Asian women } \\
>50 \text { y, African/North } \\
\text { American blacks, } \\
\text { family history of HCC) } \\
\text { - Defers to regional } \\
\text { HCC clinical practice } \\
\text { guidelines for } \\
\text { additional indications } \\
\text { in the absence of } \\
\text { cirrhosis }\end{array}$ & $\begin{array}{l}\text { - Cirrhotic patients, Child- } \\
\text { Pugh stage A and B } \\
\text { - Cirrhotic patients, Child- } \\
\text { Pugh stage C awaiting } \\
\text { liver transplantation } \\
\text { - Non-cirrhotic HBV patients } \\
\text { at intermediate or high } \\
\text { risk of HCC } \\
\text { - Non-cirrhotic patients } \\
\text { with F3 fibrosis, regardless } \\
\text { of etiology may be } \\
\text { considered based on } \\
\text { individual risk assessment }\end{array}$ \\
\hline $\begin{array}{l}\text { Screening and } \\
\text { surveillance } \\
\text { test }\end{array}$ & $\begin{array}{l}\text {-Ultrasound } \\
\text { and AFP } \\
\text { measurements } \\
\text { every } 6 \text { mo } \\
\text {-CT or MRI may } \\
\text { be used in select } \\
\text { patients with a } \\
\text { high likelihood } \\
\text { of having an } \\
\text { inadequate } \\
\text { ultrasound }\end{array}$ & $\begin{array}{l}\text { - Ultrasound and } \\
\text { AFP measurements } \\
\text { every } 6 \text { mo }\end{array}$ & $\begin{array}{l}\text { - Ultrasound with/ } \\
\text { without AFP every } \\
6 \text { mo } \\
\text { - CT or MRI may be } \\
\text { utilized in select } \\
\text { patients with a high } \\
\text { likelihood of having } \\
\text { an inadequate US or } \\
\text { with performed but } \\
\text { inadequate US }\end{array}$ & $\begin{array}{l}\text { - Ultrasound every } \\
6 \mathrm{mo}\end{array}$ & $\begin{array}{l}\text { - Ultrasound every } 6 \text { mo } \\
\text { - CT or MRI for patients } \\
\text { on waiting list for liver } \\
\text { transplantation and when } \\
\text { obesity, intestinal gas, } \\
\text { and chest wall deformity } \\
\text { prevent adequate } \\
\text { ultrasound assessment } \\
\text { - Ultrasound }<4 \text { mo interval } \\
\text { when a nodule of }<1 \mathrm{~cm} \\
\text { has been detected during } \\
\text { surveillance }\end{array}$ \\
\hline
\end{tabular}

KLCA-NCC, Korean Liver Cancer Association-National Cancer Center; APASL, Asian-Pacific Association for the Study of the Liver; AASLD, Association for the Study of Liver Diseases; LI-RADS, Liver Imaging Reporting and Data System; EASL, European Association for the Study of the Liver; HBV, hepatitis B virus; HCV, hepatitis C virus; NASH, non alcoholic steatohepatitis; PBC, primary biliary cholangitis; y, years; HCC, hepatocellular carcinoma; AFP, alpha-fetoprotein; CT, computed tomography; MRI, magnetic resonance imaging; US, ultrasonography; mo, months.

\section{IMAGING-BASED DIAGNOSIS}

Summaries of five diagnostic and staging guidelines are provided in Table 2.

\section{Target population for diagnosis}

LI-RADS defines different patient populations for surveillance and diagnosis. For the diagnosis of HCC, LI-RADS applies diagnostic imaging for HCC to positive surveillance ultrasound examinations or to incidental discovery of observations in patients at high risk for the development of HCC. More specifically, LI-RADS applies in patients at high risk for HCC, namely those with cirrhosis, chronic hepatitis B viral infection or current/prior HCC, including adult liver transplant candidates and recipients posttransplant, but does not apply to pediatric patients and cirrhosis due to vascular disorder or congenital hepatic fibrosis. ${ }^{19}$ Other systems
(AASLD, EASL, KLCA-NCC, and APASL) generally begin the diagnostic algorithm for patients with a positive screening/surveillance test. Additionally, AASLD and APASL recommend initiation of the diagnostic imaging in patients with clinical suspicion of HCC.

The differences in patient selection among guidelines are inevitable because of national and regional variations in incidence of HCC or major risk factors for HCC. The different definitions of target population affect the pre-test probability of disease and the positive and negative predictive value of tests. ${ }^{5}$

\section{Diagnostic imaging modality}

All five current systems include recommendations for multiphase CT or MRI with extracellular contrast agents (ECA) or hepatobiliary contrast agents (HBA)-enhanced MRI as first-line diagnostic imaging modalities. Of note, all guidelines except APASL do not recommend one MRI contrast agent type over the other. 
Volume 25 Number 3 September 2019

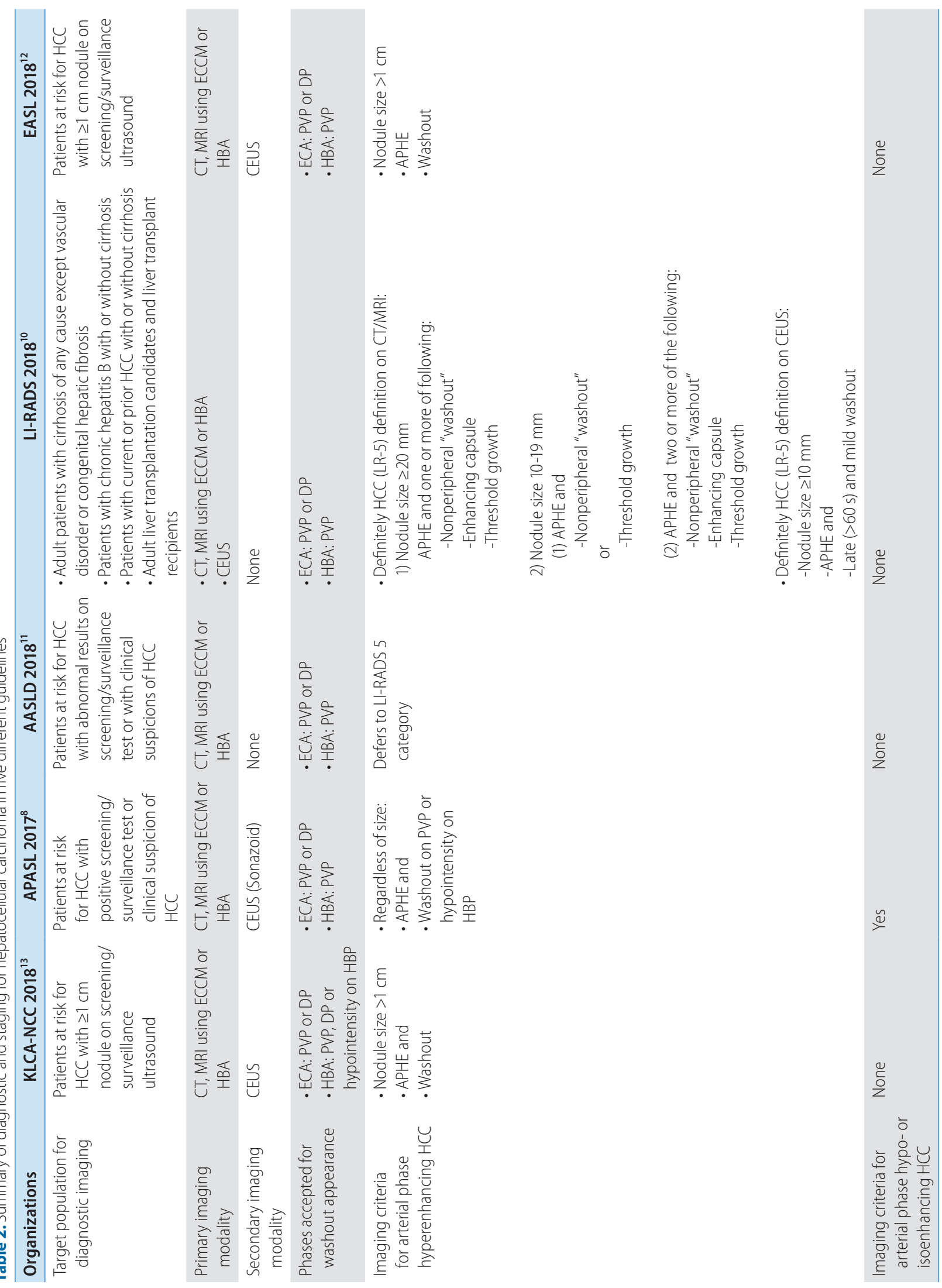




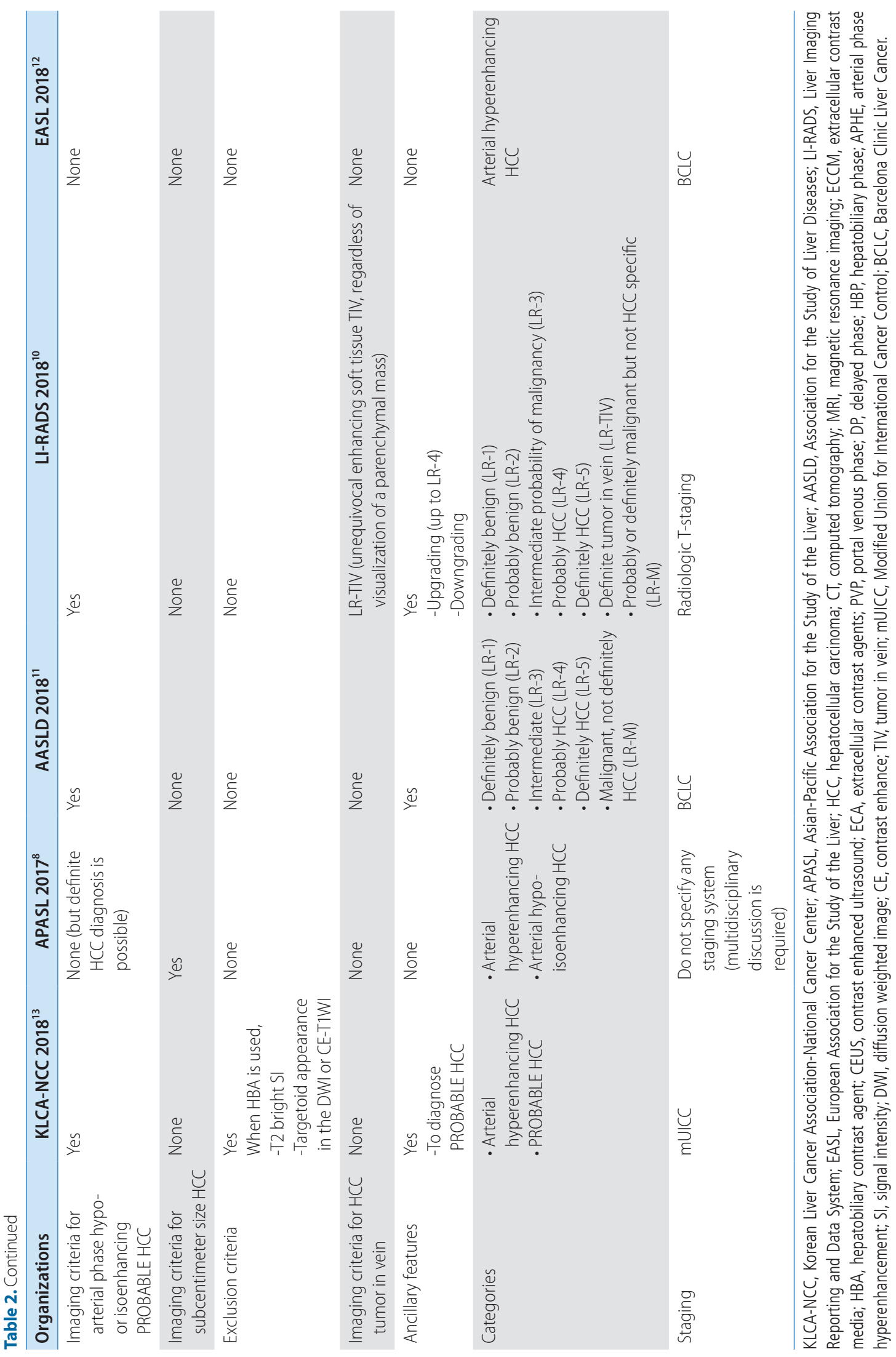


HBA include two gadolinium-based contrast agents: gadoxetate disodium (gadoxetic acid or Gd-EOB-DTPA) and gadobenate dimeglumine (Gd-BOPTA), which both have dual properties of conventional extracellular and HBA. Both agents show hepatocellular uptake via organic-anion-transporting polypeptide (OATP) transporters and biliary excretion via multidrug resistance-associated protein (MRP2) transporters. However, $50 \%$ of gadoxetate disodium compared to $5 \%$ of gadobenate dimeglumine is eliminated by biliary excretion. In general, a persistent, strong enhancement of the background liver via cellular uptake can be observed during the hepatobiliary phase (HBP) whereas premalignant or malignant hepatocellular tumors having diminished or absent transporter function show hypoenhancement. These changes in transporters of hepatocytes is shown earlier than the hemodynamic changes in hepatocarcinogenesis. ${ }^{20,21}$ Furthermore, the improved lesion-to-liver contrast during the HBP leads to higher sensitivity for the detection of HCCs than CT or MRI using extracellular contrast media. ${ }^{22}$ To acknowledge the benefits of HBAenhanced MRI, all guidelines include MRI with HBA in addition to dynamic CT or MRI with ECA as first-line diagnostic imaging tests.

For contrast-enhanced ultrasound (CEUS), LI-RADS also provides guidance for the performance and interpretation of CEUS. However, LI-RADS does not advocate for any particular imaging modality (CEUS, CT or MRI) over the other as first-line diagnostic imaging. ${ }^{23}$ Because of moderate evidence, ${ }^{12}$ the challenges of characterizing multiple nodules and comparing with $\mathrm{CT}$ or MRI examinations, EASL provided a weak recommendation in favor of using CEUS for the diagnosis of HCC, whereas KLCA-NCC and APASL recommend CEUS as second-line modality when initial first-line diagnostic imaging is inconclusive. However, AASLD has not endorsed CEUS as a diagnostic imaging for HCC.

As of now, most guidelines recommend CEUS as a secondary imaging modality, most likely due to its several potential weaknesses, i.e., the lower detection rate for washout of HCCS than with $\mathrm{CT}$ or MRI, ${ }^{24-26}$ limited capability of staging, ${ }^{24,25}$ limited performance in some patients with a poor sonic window or advanced cirrhosis, and lack of availability except in expert centers." It is controversial whether CEUS can make a specific diagnosis of HCC due to the potential risk of a misdiagnosis in the case of massforming cholangiocarcinomas (CCS) which manifest global arterial phase hyperenhancement (APHE) followed by washout at CEUS, leading to a misdiagnosis of HCC in approximately $50 \%$ of the cases. Many studies performed over the past two have proven the ability of CEUS to differentiate between HCC and CCs with different APHE characteristics, timing, and degree of washout. HCCs at
CEUS showed APHE followed by late ( $>60$ seconds) washout of a mild degree, ${ }^{27,28}$ whereas many CCS at CEUS showed APHE followed by early ( $<60$ seconds) marked washout. ${ }^{29-32}$ Recently, a large retrospective study $y^{33}$ including more than 1,000 lesions in cirrhosis, has shown that these new refined criteria of the typical HCC pattern had a positive predictive value of almost $99 \%$ for HCCs and a positive likelihood ratio of 15.5, with no false-positive diagnoses owing to CCs. In another recent prospective multicenter study ${ }^{34}$, albeit the application of the refined criteria of CEUS was unclear, CEUS showed a specificity of $92.9 \%$ vs. $76.8 \%$ and $83.2 \%$ in $10-20 \mathrm{~mm}$ nodules for $\mathrm{CT}$ and MRI, respectively. Furthermore, after a first inconclusive CT or MRI, CEUS as a second imaging technique showed the highest specificity with only a slight drop in sensitivity for 10-20 mm nodules and the highest sensitivity and specificity for 20-30 mm nodules. ${ }^{34}$

There are two types of contrast agents for CEUS: pure bloodpool contrast agents and postvascular phase agents taken up by Kupffer and/or reticuloendothelial cells. SonoVue ${ }^{\circledR}$ (sulfur hexafluoride with a phospholipid shell: Bracco SpA, Milan, Italy) and Definity ${ }^{\circledR} /$ Luminity ${ }^{\circledR}$ (octafluoropropane [perflutren] with a lipid shell: Lantheus Medical, Billerica, MA, USA) are common bloodpool agents while Sonazoid ${ }^{\circledR}$ (perfluorobutane: GE Healthcare, Chalfont St. Giles, UK) is a postvascular phase agent. Unlike SonoVue $^{\circledast}$ or Definity ${ }^{\circledR}$, Sonazoid ${ }^{\circledR}$ provides additional Kupffer phase images (usually 10 to 60 minutes after its injection) at which typical HCCs show hypoechogenicity, indicating a lack of contrast uptake corresponding to the lack of Kupffer cells and/or reticuloendothelial cells. 35,36

Both EASL and KLCA-NCC guidelines in 2018 recommend the use of intravascular contrast agents such as SonoVue ${ }^{\circledR}$ as a CEUS contrast agent for the diagnosis of hypervascular HCC. According to EASL and KLCA-NCC guidelines, to diagnose arterially hyperenhancing HCC with CEUS, the lesion larger than $1 \mathrm{~cm}$ should manifest APHE followed by late ( $>60$ seconds after injection of the contrast agent) washout of mild degree. CEUS offers several advantages over CT/MRI including the depiction of arterial hypervascularity with high temporal resolution, and better demonstration of early washout of non-HCC malignancies and of very late washout of HCC. ${ }^{37}$ These advantages of CEUS could result in high specificity when CEUS was added to either CT or MRI. ${ }^{34}$ In addition, APASL recommends usage of Sonazoid ${ }^{\circledR}$ for the diagnosis of non-hypervascular HCC (i.e., arterial phase iso- or hypoenhancing HCC on CT or MRI). ${ }^{8}$ When a non-hypervascular nodule shows hypoenhancement on HBP of gadoxetic acid-enhanced MRI, Sonazoid ${ }^{\circledR}$ enhanced ultrasonography (US) can be used as a second-line di- 
agnostic test to diagnose HCC on the basis of APHE and/or defect on defect on Kupffer phase. Until now, AASLD does not endorse the usage of CEUS in the HCC diagnostic algorithm. ${ }^{11}$

\section{Imaging criteria for arterial phase hyperenhancing $\mathrm{HCC}$}

Before we dive into the imaging criteria for the diagnosis of HCC, it is noteworthy that the AASLD 2018 practice guidance document incorporated CT/MRI LI-RADS categories into its diagnostic algorithm. " All guidelines consider the combination of APHE and washout appearance as a hallmark feature of definite HCC. There are specific differences in the definition of washout on MRI with HBA and in the size requirement for the diagnosis of definite HCC among guidelines.

When it comes to washout appearance, all guidelines require washout on either portal venous phase or delayed phase on CT/ MRI with extracellular contrast agent. However, on MRI with the HBA, AASLD, EASL, and LI-RADS strictly define washout on only portal-venous phase, whereas only KLCA-NCC encompasses washout on portal, transitional and HBP. Furthermore, when there is no washout on portal venous phases on MRI with the HBA, APASL allow hypointensity on HBP as an alternative for washout after exclusion of cavernous hemangioma. Also, the Korean guideline specifically excludes marked $\mathrm{T} 2$ hyperintensity or targetoid appearance in the diffusion weighted image or contrast enhanced T1-weighted image when HBA is used. Regarding the size of the nodule, only APASL permits the definite diagnosis of HCC regardless of size (including subcentimeter nodules) whereas other guidelines require a minimum diameter of $10 \mathrm{~mm}$ as a prerequisite for definite HCC.

Enhancing capsule appearance and threshold growth constitute major imaging features of HCC in AASLD and LI-RADS. Threshold growth is defined as a "diameter increase by at least $50 \%$ if the time interval is 6 months or less".

\section{Imaging criteria for arterial phase iso- or hypoenhancing HCC}

All current systems heavily depend on the vascular enhancement pattern appearance for imaging-based definitive diagnosis of HCC: APHE with subsequent washout. Therefore, regarding nodules with arterial phase iso- or hypoenhancement on CT or MRI, only APASL guidelines provide criteria for a definitive diagnosis of HCC whereas other guidelines do not allow the definitive diagnosis of HCC. The APASL criteria for arterial phase iso- or hypoenhancing HCC are as follows: non-APHE nodule on CT or MRI showing HBP hypointensity on HBA-enhanced MRI and hypervascularity on subsequent CEUS with Sonazoid ${ }^{\circledR}$ (defect in the Kupffer phase is not mandatory).

\section{Imaging criteria for arterial phase iso- or hypoenhancing probable HCC}

Among the five guidelines, AASLD, LI-RADS, and KLCA-NCC guidelines allow categorization of observations with arterial phase iso- or hypoenhancement as probable HCCs, whereas EASL and APASL do not allow a probable diagnosis. AASLD and LIRADS allow arterial phase iso- or hypoenhancing observations to qualify as probable HCC (designated as LI-RADS 4) with different combinations of major imaging features. For nodules with arterial phase iso- or hypoenhancement, at least one additional major feature (among nonperipheral washout, enhancing capsule or threshold growth) is required for nodules $\geq 20 \mathrm{~mm}$ and at least two additional major features are quired for nodules $<20 \mathrm{~mm}$. LIRADS also permits upgrading (up to LI-RADS 4) or downgrading LI-RADS categorization with the combination of ancillary features.

KLCA-NCC also allows categorization of arterial phase iso- or hypoenhancing nodules as probable HCC depending on the combination of ancillary imaging features. For nodules $\geq 1 \mathrm{~cm}$ in diameter with the absence of typical imaging features, the arterial phase iso- or hypoenhancing probable HCC can be diagnosed if there is at least one ancillary imaging features suggesting malignancy and at least one of those suggesting HCC appear. Ancillary imaging features suggesting malignancy include intermediate T2 hyperintensity on T2-weighted imaging, high signal intensity on diffusion-weighted imaging, and interval growth on follow-up imaging. Ancillary imaging features suggesting HCC include the presence of a capsule, mosaic appearance, nodule-in-nodule appearance, intratumoral fat, and intratumoral hemorrhage.

\section{Diagnostic scope}

The systems differ in the category of HCC. EASL guidelines address solely arterial phase hyperenhancing HCC, and two systems (AASLD and LI-RADS) address the entire spectrum of nodules and pseudolesions in patients at risk. APASL addresses not only arterial phase hyperenhancing HCC but also arterial phase iso- or hypoenhancing HCC. KLCA-NCC addresses arterial phase hyperenhancing HCC and arterial phase iso- or hypoenhancing probable HCC. 
Differences between guidelines of Western societies such as AASLD, EASL and LI-RADS, and those from Eastern societies such as KLCA-NCC and APASL are attributed to several factors such as different priorities in treatment practices. ${ }^{5,38}$ In Europe and North America, the diagnostic criteria are intentionally designed to achieve high specificity for the diagnosis of definite HCC at the expense of a decrease in sensitivity. Since patients with definite HCC may undergo liver transplantation for curative therapy based on imaging alone, high specificity was warranted to avoid falsepositive HCC diagnoses. On the other hand, in Asia, diagnostic criteria are designed to favor high sensitivity for the detection of early HCCs at the expense of a decrease in specificity. ${ }^{26,39}$ This trade-off is intentionally made to favor early treatment and access to locoregional ablative therapies such as percutaneous ethanol injection, radiofrequency ablation, and transarterial chemoembolization for the management of patients with early-stage HCCs. ${ }^{4,5,26}$

\section{Staging}

Guidelines differ in their perspective to tumor staging and favored treatment for each stage. The Barcelona Clinic Liver Cancer (BCLC) staging system is recommended by the AASLD and EASL guidelines. LI-RADS utilizes the radiologic T-staging system and KLCA-NCC guidelines endorse the Modified Union for International Cancer Control (mUICC) staging system.

\section{SUMMARIES AND KEY DIFFERENTIATING FEATURES OF EACH GUIDELINE}

\section{KLCA-NCC ${ }^{13}$}

The diagnostic algorithm for surveillance and diagnosis of HCC according to KLCA-NCC is presented in Figure 1.

\section{Target population and tests for surveillance}

The KLCA-NCC guidelines $v 2018$ recommend ultrasound and AFP measurements every 6 months for the surveillance of HCC in patients with liver cirrhosis of varying etiology and chronic HBV or HCV carrier.

Target population and imaging modalities for diagnosis First-line diagnostic imaging modalities including MRI with HBA in addition to dynamic CT or MRI with ECA should be performed in patients at risk for $\mathrm{HCC}$ with $\geq 1 \mathrm{~cm}$ nodule on surveillance ultrasound. KLCA-NCC guidelines v 2018 permit the use of intravascular contrast agents as a CEUS contrast agent for the diagnosis of hypervascular HCC.

\section{Imaging criteria for arterial phase hyperenhancing HCC}

For observations larger than $1 \mathrm{~cm}$, the combination of APHE and washout appearance is required for the diagnosis of arterial phase hyperenhancing HCC.

Regarding washout timing in HBA-enhanced MRI, the guidelines use a broad definition of "washout" as hypointensity on the portal, transitional, or HBP. Of note, considering washout up to the HBP may increase its sensitivity for the diagnosis of HCC but at the cost of lowering specificity, mainly due to the pseudowashout phenomenon (i.e., caused by parenchymal enhancement rather than tumor washout per se). ${ }^{40-42}$ In addition, the guidelines prevent the diagnosis of $\mathrm{HCC}$ for any lesion that presents either a targetoid appearance on dynamic contrast enhanced images or on diffusion weighted images or marked T2 hyperintensity when using HBA. This will prevent significant loss in specificity considering that most false-positive diagnoses of HCC resulting from the use of the HBP for determining the washout appearance were made on hemangiomas, $\mathrm{CCs}$, and combined hepatocellular- $\mathrm{CCs}{ }^{40}$ In keeping with this perspective, Joo et al. ${ }^{43}$ recently reported a sensitivity of $92.5 \%$ and specificity of $87.4 \%$ after broadening the definition of "washout" to include hypointensity on the HBP and applying the exclusion criteria.

In order to diagnose arterially hyperenhancing HCC with CEUS, the lesion larger than $1 \mathrm{~cm}$ should manifest APHE followed by late ( $>60$ seconds after injection of the contrast agent) washout of mild degree.

\section{Imaging criteria for arterial phase iso- or hypoenhancing} HCC

The guidelines do not permit the diagnosis of arterial phase isoor hypoenhancing HCC.

Imaging criteria for arterial phase iso- or hypoenhancing probable HCC

The guidelines utilize ancillary findings to diagnose probable HCCS, an approach similar to that of LI-RADS and AASLD. As a result, LI-RADS, AASLD, and KLCA-NCC use a non-binary decision algorithm covering the whole spectrum of observations seen in the liver whereas other systems including EASL and APASL use binary decisions mainly focusing on the distinction between HCCs 


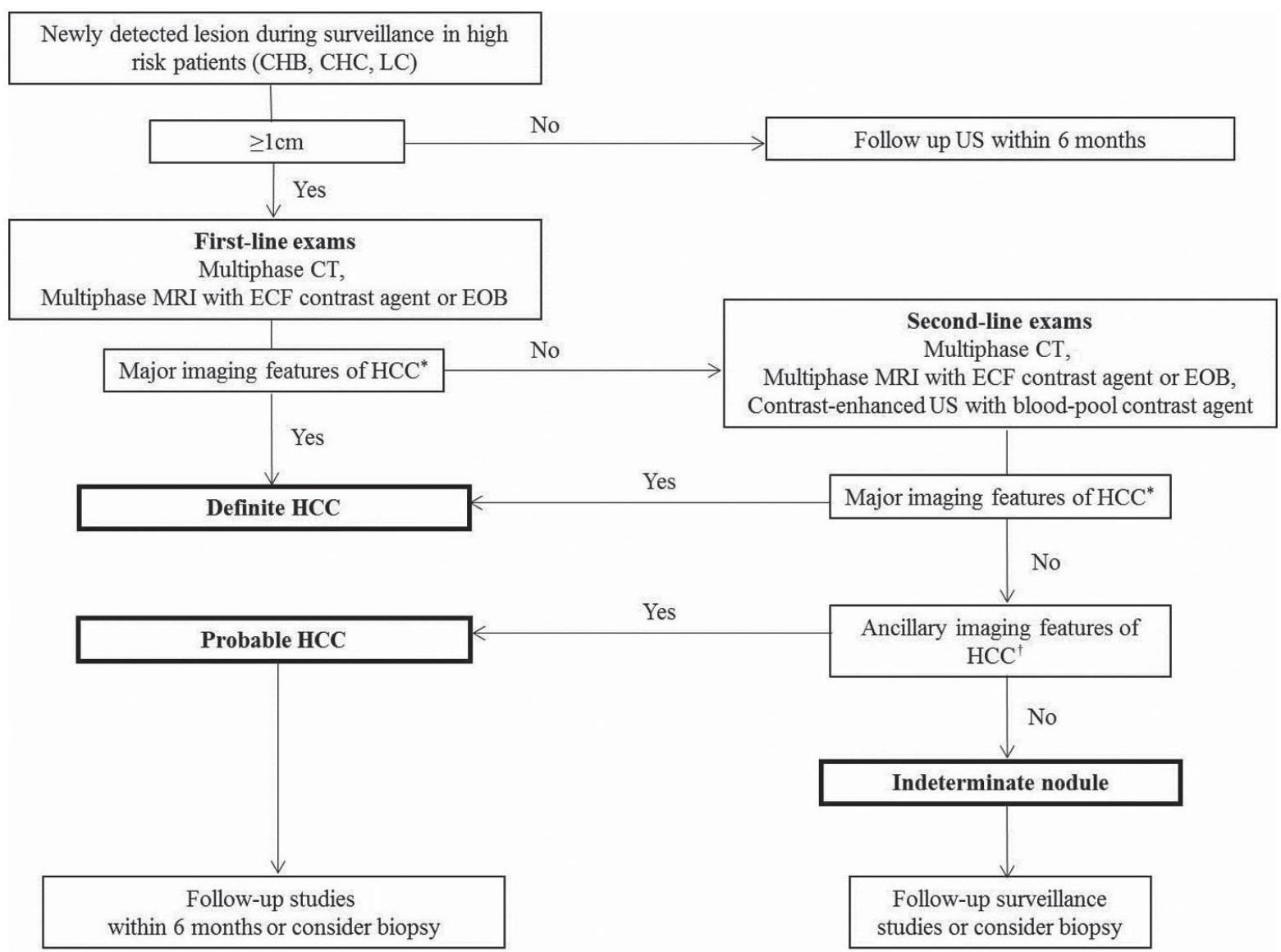

Figure 1. Diagnostic algorithm for a suspected hepatocellular carcinoma (HCC) using the new Korean Liver Cancer Association-National Cancer Center Korea practice guidelines. Adapted from KLCA-NCC guidelines. ${ }^{13}$ CHB, chronic hepatitis B virus; CHC, chronic hepatitis C virus; $L C$, liver cirrhosis; ECF, extracellular fluid. *Major imaging features of HCC include arterial hyperenhancement and the wash-out appearance during portal venous, delayed, or hepatobiliary phases on multiphasic computed tomography (CT) or magnetic resonance imaging (MRI) using extracellular contrast agents or gadoxetate disodium (EOB) in nodules $\geq 1 \mathrm{~cm}$ in diameter. However, the lesion should not show either marked T2 high signal intensity or the targetoid appearance on diffusion weighted imaging (DWI) or contrast-enhanced sequences. On contrast-enhanced ultrasonography (US) as a second line exam, major imaging features include arterial hyperenhancement and late onset ( $\geq 60$ seconds) mild wash-out; ${ }^{\dagger}$ In nodule(s) with some but not all of the aforementioned major imaging features of HCC, the category of "probable" HCC can be assigned only when the lesion fulfills at least one item from each of the following two categories of ancillary imaging features. The two categories which make up ancillary imaging features are findings favoring malignancy in general (mild-to-moderate T2 hyperintensity, restricted diffusion, hepatobiliary phase hypointensity, interval growth) and those favoring HCC in particular (non-enhancing capsule, mosaic architecture, nodule-in-nodule appearance, fat or blood products in the mass). These criteria should be applied only to a lesion which shows neither marked T2 hyperintensity nor a targetoid appearance on diffusion-weighted images or contrast-enhanced sequences.

and non HCCs.

\section{Diagnostic scope}

The guidelines provide categories including arterial hyperenhancing HCC and arterial hypo- isoenhancing probable HCC.

\section{Staging}

The guidelines utilize the mUICC staging system.

\section{APASL $^{8}$}

The diagnostic algorithm for HCC using multiple modalities according to APASL is presented in Figure 2.

\section{Target population and tests for surveillance}

The APASL guidelines recommend ultrasound and AFP measurements every 6 months for the surveillance of HCC in patients with liver cirrhosis of varying etiology (HBV, HCV, non-alcoholic 


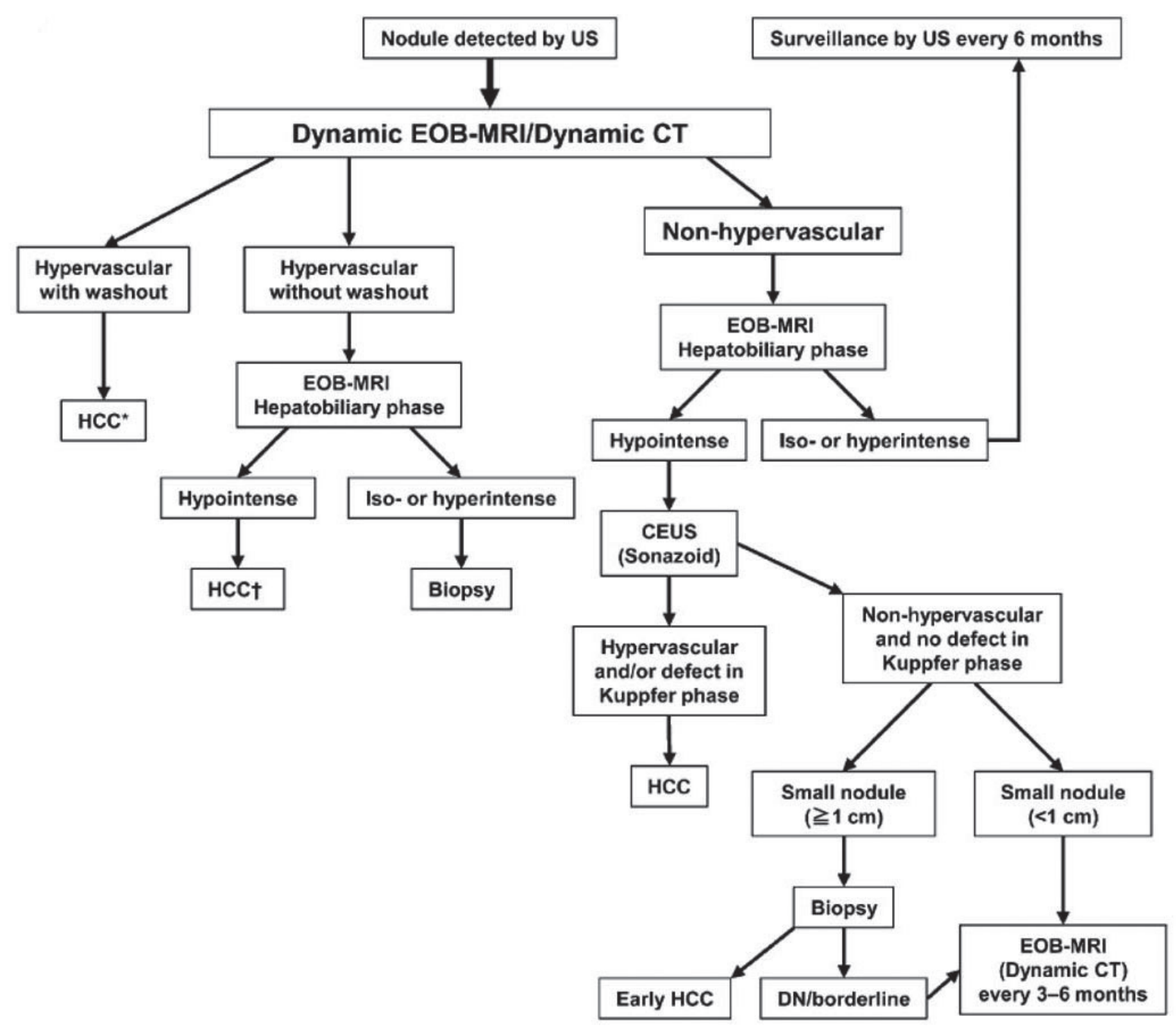

Figure 2. Diagnostic algorithm for hepatocellular carcinoma using multiple modalities according to Asian Pacific Association for the Study of the Liver (APASL). Reprint with permission from Omata et al. ${ }^{8}$ US, ultrasonography; EOB, gadoxetate disodium; CT, computed tomography; HCC, hepatocellular carcinoma; CEUS, contrast enhanced ultrasonography; DN, dysplastic nodule. * Cavernous hemangioma sometimes shows hypointensity on the equilibrium (transitional) phase of dynamic Gd-EOB DTPA magnetic resonance imaging (MRI) (pseudo-wash-out). It should be excluded by further MRI sequences and/or other imaging modalities; ${ }^{\dagger}$ Cavernous hemangioma usually shows hypointensity on the hepatobiliary phase of Gd-EOB DTPA MRI. It should be excluded by other MRI sequences and/or other imaging modalities.

steatohepatitis, genetic hemochromatosis, primary biliary cirrhosis, alpha-1 antitrypsin deficiency) and non-cirrhotic HBV patients (starting age slightly vary according to the ethnicity and family history of $\mathrm{HCC}$ ).

Target population and imaging modalities for diagnosis Regardless of the surveillance method, for patients with positive surveillance test or with clinical suspicion of HCC, the guidelines recommend the initiation of the diagnostic algorithm for HCC with first-line diagnostic imaging modalities including MRI with HBA in addition to dynamic CT or MRI with ECA. The APASL guidelines solely utilize Sonazoid ${ }^{\circledR}$ as a CEUS contrast agent for the secondary imaging modality among the five guidelines discussed in this review which will be soon later discussed.

Imaging criteria for arterial phase hyperenhancing HCC For any observations regardless of the size, the combination of APHE and washout appearance is required for the diagnosis of arterial phase hyperenhancing HCC. Regarding washout timing in HBA-enhanced MRI, APASL guidelines define "washout" as hypointensity on the portal venous phase or HBP. 


\section{Imaging criteria for arterial phase iso- or hypoenhancing} $\mathrm{HCC}$

The guidelines allow the widest diagnostic scope for HCC among the five guidelines discussed in this review article. Only the APASL guidelines permit the diagnosis of HCC on hypovascular (i.e., with arterial phase iso- or hypoenhancement) nodules on CT or MRI, regardless of the nodule size with the usage of CEUS with Sonazoid ${ }^{\circledR}$ contrast agent.

One unique feature of APASL compared to other guidelines is the usage of CEUS with Sonazoid ${ }^{\circledR}$ contrast agent. Compared to CEUS with intravascular contrast agents such as SonoVue ${ }^{\circledR}$, CEUS with Sonazoid ${ }^{\circledR}$ may achieve higher sensitivity for the detection of focal hepatic lesions due to the additional Kupffer phase. ${ }^{44}$ Furthermore, Sonazoid ${ }^{\circledR}$ with defect reperfusion imaging, which consists in reinjecting Sonazoid ${ }^{\circledR}$ and scanning areas showing a defect in the Kupffer phase, may improve the specificity for the diagnosis of HCC. ${ }^{36,45}$ However, Sonazoid ${ }^{\circledR}$ still has low specificity for the diagnosis of HCC because other hepatic lesions such as hemangiomas or CCs also lack Kupffer cells.

\section{Imaging criteria for arterial phase iso- or hypoenhancing probable HCC}

The guidelines do not permit the diagnosis of arterial phase isoor hypoenhancing probable HCC.

\section{Diagnostic scope}

The guidelines provide categories including arterial hyperenhancing HCC and arterial hypo- isoenhancing HCC.

\section{Staging}

The guidelines do not specify the staging system and advocate multidisciplinary decision regarding treatment plan such as liver resection.

\section{SURVEILLANCE}
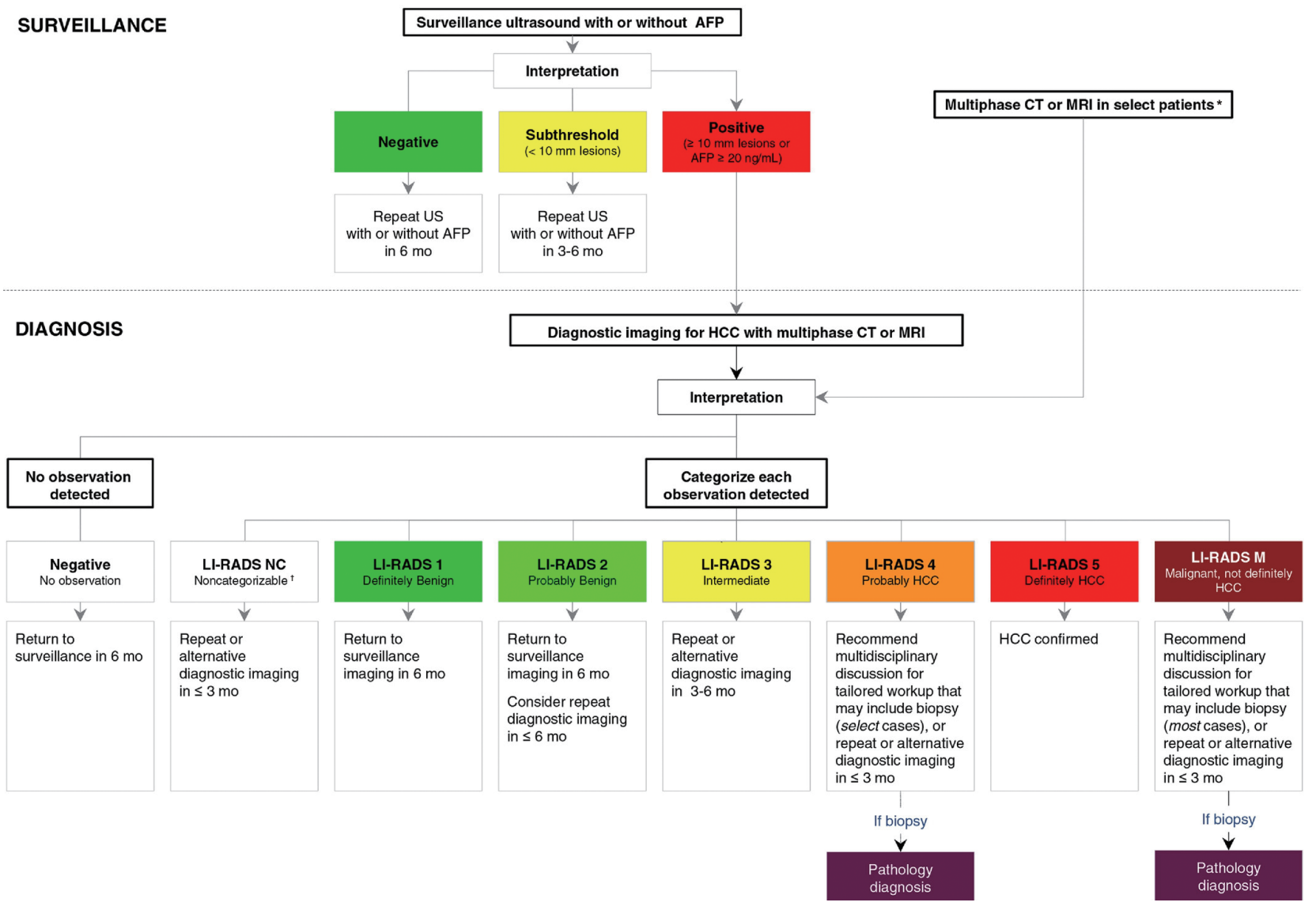

Figure 3. Diagnostic algorithm for surveillance and diagnosis of hepatocellular carcinoma (HCC) according to American Association for the Study of Liver Disease (AASLD). Reprint with permission from Marrero et al." AFP, alphafeto protein; US, ultrasonography; mo, months; CT, computed tomography; MRI, magnetic resonance imaging; LI-RADS, Liver Imaging Reporting and Data System; NC, noncategorizable; M, malignancy (not necessarily HCC). *Some high-risk patients may undergo multiphase CT or MRI for HCC surveillance (depending on patient body habitus, visibility of liver at ultrasound, being on the transplant waiting list and other factors); ${ }^{\dagger}$ These are due to technical problem such as image omission or severe degradation. 


\section{AASLD $^{11}$}

The diagnostic algorithm for surveillance and diagnosis of HCC according to AASLD is presented in Figure 3.

\section{Target population and tests for surveillance}

The AASLD guidelines recommend ultrasound with or without AFP every 6 months for the surveillance of HCC in patients with liver cirrhosis of varying etiology (HBV, HCV, primary biliary cirrhosis, genetic hemochromatosis, alpha-1 antitrypsin deficiency) and non-cirrhotic HBV patients (starting age vary according to the ethnicity and family history of HCC). CT or MRI may be utilized in select patients with a high likelihood of having an inadequate US or with performed but inadequate US examination.

\section{Target population and imaging modalities for diagnosis}

For patients with positive surveillance test or with clinical suspicion of HCC, the guidelines recommend the initiation of the diagnostic algorithm for HCC. A key change in the 2018 Practice Guidance document by the AASLD is the integration of LI-RADS into its diagnostic algorithm. AASLD recognizes that LI-RADS categories reflect the probability of HCC and of other malignancy and reflect the cumulative incidences of progression of untreated observations. For example, LI-RADS 3 observations have average possibility of $33 \%$ for $\mathrm{HCC}^{46-48}$ with $6-15 \%$ progression into HCC or other malignancy by 24 months. ${ }^{49-51}$ LI-RADS 4 lesion has an average probability of HCC of $80 \%{ }^{46-48}$ with $46-68 \%$ progression into HCC or other malignancy by 24 months. ${ }^{49-51}$ The AASLD surveillance and diagnostic algorithm also links LI-RADS categories with management recommendations.

CEUS was previously recommended in the 2005 AASLD guidelines as part of the diagnostic algorithm. ${ }^{52}$ However, CEUS were not recommended in the 2011 or 2018 versions and not included in the AASLD diagnostic algorithm, unlike the four other guidelines which either include CEUS as a first-line or second-line imaging modality. The AASLD guidance document cites concerns mentioned in a meta-analysis, ${ }^{53}$ including: absence of a large-scale study, potential selection bias for patients with adequate quality ultrasounds, lack of generalizability of studies in Asia versus Western countries, and operator dependency.

\section{Imaging criteria for arterial phase hyperenhancing HCC}

LI-RADS category 5 is equivalent to the arterial phase hyperenhancing HCC. Defer to LI-RADS section below for the specific diagnostic criteria.
Imaging criteria for arterial phase iso- or hypoenhancing $\mathrm{HCC}$

The guidelines do not permit the diagnosis of arterial phase isoor hypoenhancing HCC.

Imaging criteria for arterial phase iso- or hypoenhancing probable HCC

The guidelines allow arterial phase iso- or hypoenhancing observations to qualify as probable HCC (designated as LI-RADS 4) with different combinations of major imaging features. Defer to "diagnostic scope" on LI-RADS section below for further details.

\section{Diagnostic scope}

Defer to "diagnostic scope" on LI-RADS section below for further details.

\section{Staging}

The guidelines recommend the BCLC staging system.

\section{LI-RADS $^{10}$}

The CT/MRI diagnostic algorithm of HCC according to LI-RADS is presented in Figure 4.

\section{Target population and tests for surveillance}

The LI-RADS guidelines recommend ultrasound every 6 months for the surveillance of HCC in patients with liver cirrhosis of any etiology and non-cirrhotic HBV patients (Asian male $>40$ years old, Asian female $>50$ years old, African or North American Blacks with HBV, family history of HBV). The guidelines also defer to regional HCC clinical practice guidelines for additional indications in the absence of cirrhosis.

\section{Target population and imaging modalities for diagnosis}

The target population for the initiation of the diagnostic algorithm for HCC is broader compared to those for the surveillance; adult patients with cirrhosis of any cause except vascular disorder or congenital hepatic fibrosis, patients with chronic hepatitis B with or without cirrhosis, patients with current or prior HCC with or without cirrhosis, and adult liver transplantation candidates and liver transplant recipient.

First-line diagnostic imaging modalities includes not only dynamic CT or MRI with ECA or with HBA, but also CEUS. LI-RADS is the only guideline which permits the diagnosis of $\mathrm{HCC}$ with CEUS as the initial imaging modality. HCC can be diagnosed defi- 
nitely on CEUS if observations equal or larger than $1 \mathrm{~cm}$ show APHE followed by late (>60 seconds) and mild washout (CEUS LR-5). Observations that show rim APHE, early ( $<60$ seconds) washout or marked washout indicating probably or definitely malignant observations, but not HCC specific are assigned the CEUS LR-M category. Although CEUS has many advantages such as real-time imaging with high temporal resolution, safe and nonvisualization of vascular pseudolesions, LI-RADS CEUS also acknowledges its limitations: has fewer ancillary features, permits characterization of a limited number of targeted observations per examination (preferably those that are visible on precontrast US) and hence is not suitable for staging.
Imaging criteria for arterial phase hyperenhancing HCC

LI-RADS category 5 is equivalent to the arterial phase hyperenhancing HCC and the diagnostic criteria as follows:

Nodule size $\geq \mathbf{2 0} \mathbf{~ m m}$ : -APHE and one or more of following: nonperipheral "washout", enhancing "capsule", or threshold growth.

Nodule size 10-19 mm: -APHE and nonperipheral washout or threshold growth.

-APHE and two or more of the following: nonperipheral washout, enhancing capsule, or threshold growth.

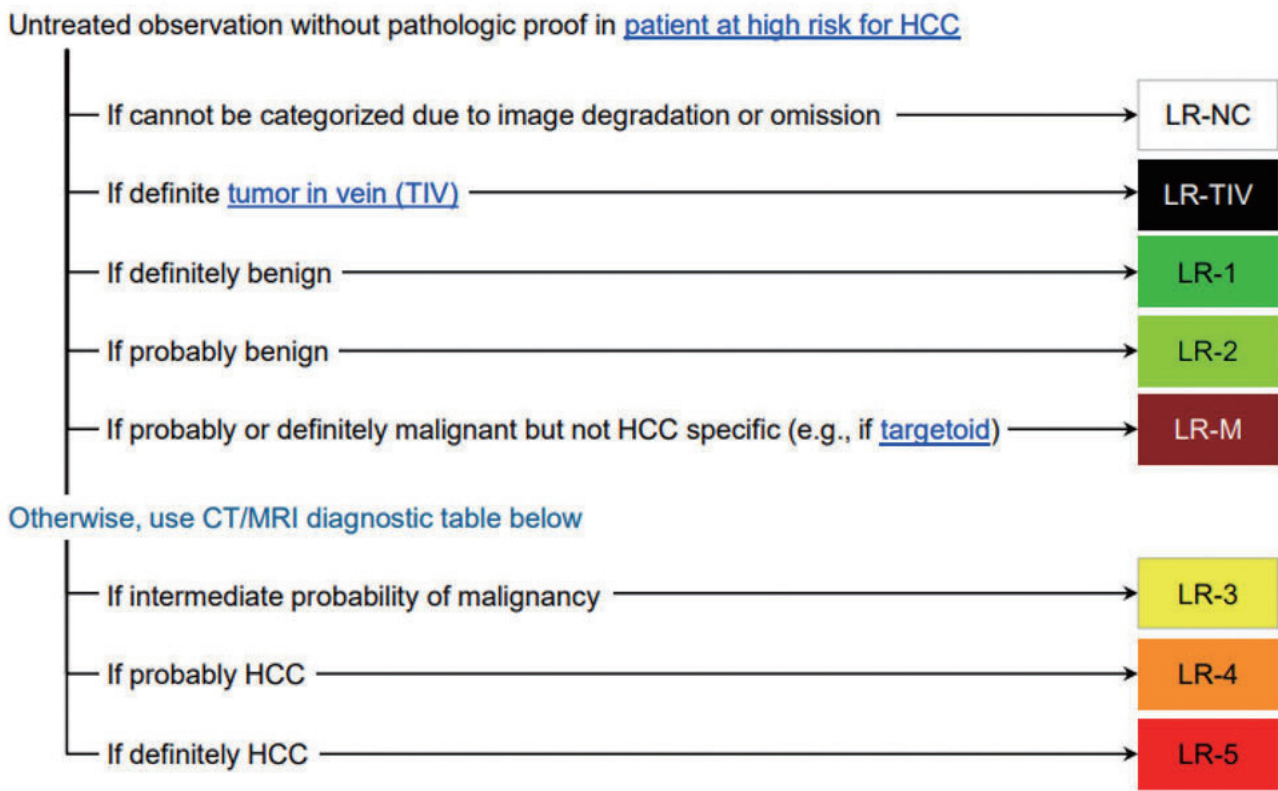

\section{CT/MRI Diagnostic Table}

\begin{tabular}{|c|c|c|c|c|c|c|}
\hline \multirow{2}{*}{\multicolumn{2}{|c|}{$\begin{array}{l}\text { Arterial phase hyperenhancement (APHE) } \\
\text { Observation size }(\mathrm{mm})\end{array}$}} & \multicolumn{2}{|c|}{ No APHE } & \multicolumn{3}{|c|}{ Nonrim APHE } \\
\hline & & $<20$ & $\geq 20$ & $<10$ & $10-19$ & $\geq 20$ \\
\hline \multirow{3}{*}{$\begin{array}{l}\text { Count additional major features: } \\
\text { - Enhancing "capsule" } \\
\text { - Nonperipheral "washout" } \\
\text { - Threshold growth }\end{array}$} & None & LR-3 & LR-3 & LR-3 & LR-3 & LR-4 \\
\hline & One & LR-3 & LR-4 & LR-4 & $\begin{array}{l}\text { LR-4 } \\
\text { LR- }\end{array}$ & LR-5 \\
\hline & $\geq$ Two & LR-4 & LR-4 & LR-4 & LR-5 & LR-5 \\
\hline
\end{tabular}

\section{LR-4}

Observations in this cell are categorized based on one additional major feature:

- LR-4 - if enhancing "capsule"

- LR-5 - if nonperipheral "washout" OR threshold growth

Figure 4. The computed tomography (CT)/magnetic resonance imaging (MRI) diagnostic algorithm of hepatocellular carcinoma (HCC) according to Liver Imaging Reporting and Data System (LI-RADS) v 2018. Reprint with permission from American College of Radiology. ${ }^{10}$ LR, lirads; NC, noncategorizable; TIV, tumor in vein; $M$, malignancy (not necessarily HCC). 


\section{Imaging criteria for arterial phase iso- or hypoenhancing} HCC

The guidelines do not permit the diagnosis of definite HCC (designated as (R-5) with arterial phase iso- or hypoenhancing observations.

\section{Imaging criteria for arterial phase iso- or hypoenhancing probable HCC}

The guidelines allow arterial phase iso- or hypoenhancing observations to qualify as probable HCC (designated as LR-4) with different combinations of major imaging features.

\section{Diagnostic scope}

LI-RADS is a comprehensive system designed to standardize the acquisition, interpretation and reporting of CT, MRI and CEUS examination of the liver in patients at risk for HCC and to provide precisely defined terminology, an illustrative atlas, a diagnostic algorithm, and guidance for appropriate imaging technique. Unlike other systems, LI-RADS addresses the full spectrum of lesions and pseudolesions encountered on imaging in patients at risk for HCC. Observations are classified into categories that reflect their relative probability benignity or malignancy (ranging from LR-1 to LR5) or malignancy, not specific for HCC (LR-M) on the basis of CT, MRI, or CEUS findings. ${ }^{24,54}$

LI-RADS also incorporates ancillary imaging features that modulate the likelihood of HCC. Radiologists may, at their discretion, apply ancillary features that favor HCC to upgrade the category by one or more categories (up to but not beyond LR-4) or apply an-

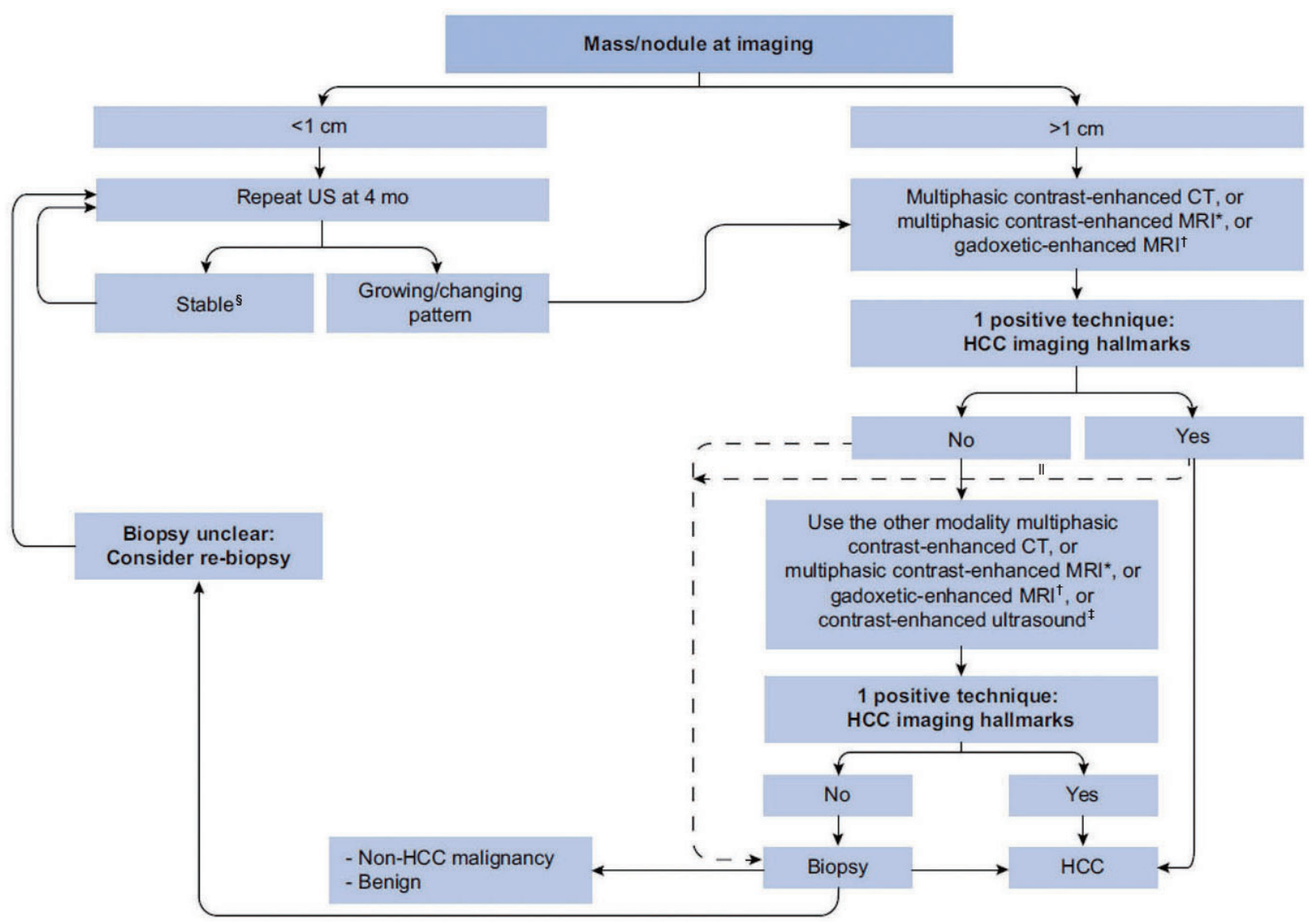

Figure 5. Diagnostic algorithm and recall policy in cirrhotic liver according to European Association for the Study of the Liver (EASL). Reprint with permission from EASL.' ${ }^{12}$ US, ultrasonography; mo, months; CT, computed tomography; MRI, magnetic resonance imaging; HCC, hepatocellular carcinoma. *Using extracellular magnetic resonance contrast agents or gadobenate dimeglumine; ${ }^{\dagger} U$ sing the following diagnostic criteria: arterial phase hyperenhancement (APHE) and washout on the portal venous phase; ${ }^{\ddagger}$ Using the following diagnostic criteria: APHE and mild washout after 60 seconds; ${ }^{\circledR}$ Lesion $<1 \mathrm{~cm}$ stable for 12 months (three controls after four months) can be shifted back to regular 6 months surveillance; "Optional for centre-based programmes. 
cillary features that may favor benignity to downgrade the category by one or more categories.

\section{Staging}

The guidelines recommend radiologic T-staging.

\section{EASL}

The diagnostic algorithm and recall policy in cirrhotic liver according to EASL is presented in Figure 5.

\section{Target population and tests for surveillance}

The EASL guidelines recommend ultrasound every 6 months for the surveillance of HCC in cirrhotic patients with Child-Pugh stage $A / B$ or with stage $C$ awaiting liver transplantation and in non-cirrhotic patients with HBV infection at intermediate or high risk for HCC or with F3 fibrosis regardless of etiology. Ultrasound less than 4-month interval is recommended when a sub centimeter nodule has been detected. CT or MRI may also be utilized for patients on waiting list for liver transplantation and for patients with inadequate ultrasound assessment is expected due to obesity, internal gas, and chest wall deformity.

\section{Target population and imaging modalities for diagnosis}

First-line diagnostic imaging modalities including MRI with HBA in addition to dynamic CT or MRI with ECA should be performed in patients at risk for $\mathrm{HCC}$ with $\geq 1 \mathrm{~cm}$ nodule on surveillance ultrasound. EASL v 2018 newly permitted the use of CEUS as a secondary imaging modality for the diagnosis of hypervascular HCC.

\section{Imaging criteria for arterial phase hyperenhancing HCC}

For observations larger than $1 \mathrm{~cm}$, the combination of APHE and washout appearance is required for the diagnosis of arterial phase hyperenhancing HCC. Contrary to the KLCA-NCC guidelines v 2018, the EASL guidelines only allow a narrow definition of "washout" as hypointensity only on the portal venous phase in HBA-enhanced MRI.

\section{Imaging criteria for arterial phase iso- or hypoenhancing} HCC

The guidelines do not permit the diagnosis of arterial phase isoor hypoenhancing HCC.

\section{Imaging criteria for arterial phase iso- or hypoenhancing probable HCC}

The guidelines do not permit the diagnosis of arterial phase isoor hypoenhancing probable HCC.

\section{Diagnostic scope}

Among the five guidelines discussed in this review article, EASL provides the narrowest diagnostic scope for $\mathrm{HCC}$ by using the following diagnostic criteria for nodules $\geq 1 \mathrm{~cm}$ : arterial phase hyperenhancement and washout on the portal venous phase or delayed phase on CT and MRI studies using ECA or washout on the portal venous phase on HBA-enhanced MRI. The guidelines do not approve the diagnosis of HCC or probable HCC for nodules with isoor hypovascularity and does not apply any ancillary features that may modulate the likelihood of HCC.

\section{Staging \\ The guidelines utilize the BCLC staging system.}

\section{LIMITATIONS AND FUTURE DIRECTIONS}

There are some limitations of the current systems that need to be addressed.

First, the systems vary reflecting differences in technology, cultural factors, clinical practice patterns, and management strategies. Although these differences among the systems may be unavoidable, radiologists must adapt to the difference between these systems. The current absence of unification and standardization of HCC imaging systems poses many disadvantages such as limitation on the development of registries, sharing of data and adoption of scientific advances developed from other systems; classification of the same nodule differently depending on the diagnostic systems; and hindrance of knowledge dissemination from other institutions adopting different systems. The unification and standardization of HCC imaging systems may be anticipated after the identification of potential barriers and the harmonization of different aspects of diverse systems.

Second, a definite diagnosis of HCC is not allowed for small $(<1 \mathrm{~cm})$ nodules or arterial iso- or hypoenhancing HCCs in most guidelines except APASL. Indeed, rendering a reliable diagnosis for small nodules $(<1 \mathrm{~cm})$ on CT or MRI as HCC may be difficult due to the low probability of $\mathrm{HCC}^{22,38} \mathrm{Also}$, for the sake of high specificity in the diagnosis of $\mathrm{HCC}$, encompassing atypical and hypovascular lesions for the diagnosis of HCC may pose challenges. However, 
considering the high progression rate (approximately more than $90 \%$ ) of subcentimeter hypervascular nodules with typical HCC imaging findings on gadoxetic acid enhanced MRI to typical $\mathrm{HCCS}^{55,56}$ and improved prognosis of very early stage HCCs compared to early-stage $\mathrm{HCCs}$, 38,57 the diagnosis of subcentimeter HCCs can be of clinical benefit. Furthermore, instead of excluding the diagnosis of HCC to all nodules with arterial iso- or hypoenhancing nodules on $\mathrm{CT}$ or MRI, additional investigation using other imaging modalities may be warranted to avoid missing HCCs. This is best illustrated by APASL which recommends using CEUS with Sonazoid ${ }^{\circledast}$ to characterize hypovascular nodules on initial CT or MRI.

Third, although there has been a trend toward the use of CEUS as a secondary modality, as endorsed by EASL, KLCA-NCC, and APASL, there is scarce evidence justifying the selection of the most appropriate second-line imaging modality after an initial inconclusive examination and comparative evidence between different CEUS contrast agents. Only APASL accepts the use of Sonazoid $^{\circledR}$ whereas most other guidelines including EASL, LI-RADS, and KLCA-NCC accept the use of pure blood-pool contrast agents such as SonoVue ${ }^{\circledR}$. Therefore, further prospective studies involving a larger number of patients are warranted to confirm the role of CEUS as the most effective secondary modality and head-tohead studies are required to compare the diagnostic performance between CEUS agents.

Lastly, additional imaging methods other than dynamic imaging, such as diffusion-weighted imaging or HBP can be included in the diagnostic criteria to compensate the imperfect sensitivity and specificity of the current noninvasive diagnostic criteria. There is a trend for the adoption of these additional imaging methods. For example, LI-RADS and KLCA-NCC guidelines include restricted diffusion as an ancillary feature favoring malignancy in general, and marked $\mathrm{T} 2$ hyperintensity as an ancillary feature favoring benignity. Furthermore, HBA-enhanced MRI may further be utilized widely considering the potential benefit of the characterization of lesions on the basis of both vascularity and hepatocellular uptake. $^{58}$

\section{CONCLUSION}

Several scientific organizations have proposed systems that incorporate imaging for surveillance and diagnosis for HCC. The designs and component of these systems differ considerably reflecting different target populations, resources, and treatment practices. In 2018 particular, LI-RADS, AASLD, EASL, and KLCANCC have been updated. Key changes include wider recognition of hepatobiliary agents and CEUS, expecting the increased role in both modalities in the noninvasive diagnosis of $\mathrm{HCC}$ in the years to come. Integration of LI-RADS into the AASLD practice guidance also announces future harmonization and unification of diagnostic imaging systems to facilitate knowledge dissemination. Yet, several issues remain to be solved regarding the diversity of the systems, diagnosis of subcentimeter HCCs and hypovascular HCCs, evaluation of CEUS as an appropriate second-line modality, the head-to-head comparaison of different CEUS contrast agents (SonoVue $^{\circledR}$ and Sonazoid ${ }^{\circledR}$ ), and adoption of ancillary imaging features seen not assessed on dynamic imaging. In the near future, these issues can be clarified on the basis of large-scale data-driven evidence.

\section{Authors' contribution}

KTH: Conception or design of the work, Data collection, Data analysis and interpretation, Drafting the article, Critical revision of the article, and Final approval of the version.

KSY: Critical revision of the article.

TA: Critical revision of the article, and Final approval of the version.

LJM: Conception or design of the work, Critical revision of the article, and Final approval of the version.

\section{Conflicts of Interest}

Authors declare no conflicts of interest.

\section{REFERENCES}

1. Choo SP, Tan WL, Goh BKP, Tai WM, Zhu AX. Comparison of hepatocellular carcinoma in Eastern versus Western populations. Cancer 2016;122:3430-3446.

2. Global Burden of Disease Liver Cancer Collaboration, Akinyemiju T, Abera S, Ahmed M, Alam N, Alemayohu MA, et al. The burden of primary liver cancer and underlying etiologies from 1990 to 2015 at the global, regional, and national level: results from the global burden of disease study 2015. JAMA Oncol 2017;3:1683-1691.

3. Shields A, Reddy KR. Hepatocellular carcinoma: current treatment strategies. Curr Treat Options Gastroenterol 2005;8:457-466.

4. Heimbach JK, Kulik LM, Finn RS, Sirlin CB, Abecassis MM, Roberts $L R$, et al. AASLD guidelines for the treatment of hepatocellular carcinoma. Hepatology 2018;67:358-380.

5. Tang A, Cruite I, Mitchell DG, Sirlin CB. Hepatocellular carcinoma 
imaging systems: why they exist, how they have evolved, and how they differ. Abdom Radiol (NY) 2018;43:3-12.

6. Tang A, Cruite I, Sirlin CB. Toward a standardized system for hepatocellular carcinoma diagnosis using computed tomography and MRI. Expert Rev Gastroenterol Hepatol 2013;7:269-279.

7. Gavriilidis P, Roberts KJ, Askari A, Sutcliffe RP, Huo TL, Liu PH, et al. Evaluation of the current guidelines for resection of hepatocellular carcinoma using the Appraisal of Guidelines for Research and Evaluation II instrument. J Hepatol 2017;67:991-998.

8. Omata M, Cheng AL, Kokudo N, Kudo M, Lee JM, Jia J, et al. AsiaPacific clinical practice guidelines on the management of hepatocelIular carcinoma: a 2017 update. Hepatol Int 2017;11:317-370.

9. American College of Radiology (ACR). Liver Imaging Reporting and Data System version 2017. ACR web site, <https://www.acr.org/ Quality-Safety/Resources/LIRADS/LIRADS-v2017>. Accessed 31 Oct 2018.

10. American College of Radiology (ACR). Liver Imaging Reporting and Data System version 2018. ACR web site, <https://www.acr.org/ Clinical-Resources/Reporting-and-Data-Systems/LI-RADS>. Accessed 31 Oct 2018.

11. Marrero JA, Kulik LM, Sirlin C, Zhu AX, Finn RS, Abecassis MM, et al. Diagnosis, staging and management of hepatocellular carcinoma: 2018 practice guidance by the American Association for the Study of Liver Diseases. Hepatology 2018;68:723-750.

12. European Association for the Study of the Liver. EASL Clinical Practice Guidelines: management of hepatocellular carcinoma. J Hepatol 2018;69:182-236.

13. Korean Liver Cancer Association-National Cancer Center (KLCANCC). 2018 KLCA-NCC Korea Practice Guideline for the Management of Hepatocellular Carcinoma. KLCA-NCC web site, <http:// livercancer.or.kr/study/guidelines.php>. Accessed 31 Oct 2018.

14. Cruite I, Tang A, Sirlin CB. Imaging-based diagnostic systems for hepatocellular carcinoma. AJR Am J Roentgenol 2013;201:41-55.

15. American College of Radiology (ACR). Ultrasound LI-RADS(R) V2017. ACR web site, <https://www.acr.org/Clinical-Resources/Reportingand-Data-Systems/LI-RADS/Ultrasound-LI-RADS-v2017>. Accessed 31 Oct 2018.

16. Dulku G, Dhillon R, Goodwin M, Cheng W, Kontorinis N, Mendelson $R$. The role of imaging in the surveillance and diagnosis of hepatocellular cancer. J Med Imaging Radiat Oncol 2017;61:171-179.

17. Singal A, Volk ML, Waljee A, Salgia R, Higgins P, Rogers MA, et al. Meta-analysis: surveillance with ultrasound for early-stage hepatocellular carcinoma in patients with cirrhosis. Aliment Pharmacol Ther 2009;30:37-47.

18. Tzartzeva K, Obi J, Rich NE, Parikh ND, Marrero JA, Yopp A, et al. Surveillance imaging and alpha fetoprotein for early detection of hepatocellular carcinoma in patients with cirrhosis: a meta-analysis. Gastroenterology 2018;154:1706-1718.e1.
19. Chernyak V, Santillan CS, Papadatos D, Sirlin CB. LI-RADS(R) algorithm: CT and MRI. Abdom Radiol (NY) 2018;43:111-126.

20. Kitao A, Matsui O, Yoneda N, Kozaka K, Shinmura R, Koda W, et al. The uptake transporter OATP8 expression decreases during multistep hepatocarcinogenesis: correlation with gadoxetic acid enhanced MR imaging. Eur Radiol 2011;21:2056-2066.

21. Kogita S, Imai Y, Okada M, Kim T, Onishi H, Takamura M, et al. GdEOB-DTPA-enhanced magnetic resonance images of hepatocellular carcinoma: correlation with histological grading and portal blood flow. Eur Radiol 2010;20:2405-2413.

22. Lee YJ, Lee JM, Lee JS, Lee HY, Park BH, Kim YH, et al. Hepatocellular carcinoma: diagnostic performance of multidetector $\mathrm{CT}$ and MR imaging-a systematic review and meta-analysis. Radiology 2015;275:97-109.

23. Kim TK, Noh SY, Wilson SR, Kono Y, Piscaglia F, Jang HJ, et al. Contrast-enhanced ultrasound (CEUS) liver imaging reporting and data system (LI-RADS) 2017 - a review of important differences compared to the CT/MRI system. Clin Mol Hepatol 2017;23:280289.

24. Leoni S, Piscaglia F, Granito A, Borghi A, Galassi M, Marinelli S, et al. Characterization of primary and recurrent nodules in liver cirrhosis using contrast-enhanced ultrasound: which vascular criteria should be adopted? Ultraschall Med 2013;34:280-287.

25. Furlan A, Marin D, Cabassa P, Taibbi A, Brunelli E, Agnello F, et al. Enhancement pattern of small hepatocellular carcinoma (HCC) at contrast-enhanced US (CEUS), MDCT, and MRI: intermodality agreement and comparison of diagnostic sensitivity between 2005 and 2010 American Association for the Study of Liver Diseases (AASLD) guidelines. Eur J Radiol 2012;81:2099-2105.

26. Forner A, Vilana R, Ayuso C, Bianchi L, Solé M, Ayuso JR, et al. Diagnosis of hepatic nodules $20 \mathrm{~mm}$ or smaller in cirrhosis: prospective validation of the noninvasive diagnostic criteria for hepatocellular carcinoma. Hepatology 2008;47:97-104.

27. Piscaglia F, Kudo M, Han KH, Sirlin C. Diagnosis of hepatocellular carcinoma with non-invasive imaging: a plea for worldwide adoption of standard and precise terminology for describing enhancement criteria. Ultraschall Med 2017;38:9-11.

28. Piscaglia F, Wilson SR, Lyshchik A, Cosgrove D, Dietrich CF, Jang HJ, et al. American College of Radiology Contrast Enhanced Ultrasound Liver Imaging Reporting and Data System (CEUS LI-RADS) for the diagnosis of hepatocellular carcinoma: a pictorial essay. Ultraschall Med 2017;38:320-324.

29. Chen LD, Xu HX, Xie XY, Xie XH, Xu ZF, Liu GJ, et al. Intrahepatic cholangiocarcinoma and hepatocellular carcinoma: differential diagnosis with contrast-enhanced ultrasound. Eur Radiol 2010;20:743753.

30. Claudon M, Dietrich CF, Choi BI, Cosgrove DO, Kudo M, Nolsøe $\mathrm{CP}$, et al. Guidelines and good clinical practice recommendations 
for contrast enhanced ultrasound (CEUS) in the liver--update 2012: a WFUMB-EFSUMB initiative in cooperation with representatives of AFSUMB, AIUM, ASUM, FLAUS and ICUS. Ultraschall Med 2013;34:11-29.

31. Wildner D, Bernatik T, Greis C, Seitz K, Neurath MF, Strobel D. CEUS in hepatocellular carcinoma and intrahepatic cholangiocellular carcinoma in 320 patients - early or late washout matters: a subanalysis of the DEGUM multicenter trial. Ultraschall Med 2015;36:132-139.

32. Wildner D, Pfeifer L, Goertz RS, Bernatik T, Sturm J, Neurath MF, et al. Dynamic contrast-enhanced ultrasound (DCE-US) for the characterization of hepatocellular carcinoma and cholangiocellular carcinoma. Ultraschall Med 2014;35:522-527.

33. Terzi $\mathrm{E}$, lavarone $\mathrm{M}$, Pompili $\mathrm{M}$, Veronese $\mathrm{L}$, Cabibbo $\mathrm{G}$, Fraquelli $\mathrm{M}$, et al. Contrast ultrasound LI-RADS LR-5 identifies hepatocellular carcinoma in cirrhosis in a multicenter restropective study of 1,006 nodules. J Hepatol 2018;68:485-492.

34. Aubé C, Oberti F, Lonjon J, Pageaux G, Seror O, N'Kontchou G, et al. EASL and AASLD recommendations for the diagnosis of HCC to the test of daily practice. Liver Int 2017;37:1515-1525.

35. Bota S, Piscaglia F, Marinelli S, Pecorelli A, Terzi E, Bolondi L. Comparison of international guidelines for noninvasive diagnosis of hepatocellular carcinoma. Liver Cancer 2012;1:190-200.

36. Kudo M. Defect reperfusion imaging with Sonazoid(R): a breakthrough in hepatocellular carcinoma. Liver Cancer 2016;5:1-7.

37. Jang HJ, Kim TK, Burns PN, Wilson SR. CEUS: an essential component in a multimodality approach to small nodules in patients at high-risk for hepatocellular carcinoma. Eur J Radiol 2015;84:16231635.

38. Farinati F, Sergio A, Baldan A, Giacomin A, Di Nolfo MA, Del Poggio $P$, et al. Early and very early hepatocellular carcinoma: when and how much do staging and choice of treatment really matter? A multi-center study. BMC Cancer 2009;9:33.

39. Wald C, Russo MW, Heimbach JK, Hussain HK, Pomfret EA, Bruix J. New OPTN/UNOS policy for liver transplant allocation: standardization of liver imaging, diagnosis, classification, and reporting of hepatocellular carcinoma. Radiology 2013;266:376-382.

40. Joo I, Lee JM, Lee DH, Jeon JH, Han JK, Choi BI. Noninvasive diagnosis of hepatocellular carcinoma on gadoxetic acid-enhanced MRI: can hypointensity on the hepatobiliary phase be used as an alternative to washout? Eur Radiol 2015;25:2859-2868.

41. Choi SH, Byun JH, Lim YS, Yu E, Lee SJ, Kim SY, et al. Diagnostic criteria for hepatocellular carcinoma $3 \mathrm{~cm}$ with hepatocytespecific contrast-enhanced magnetic resonance imaging. J Hepatol 2016;64:1099-1107.

42. Kierans AS, Kang SK, Rosenkrantz AB. The diagnostic performance of dynamic contrast-enhanced MR imaging for detection of small hepatocellular carcinoma measuring up to $2 \mathrm{~cm}$ : a meta-analysis. Radiology 2016;278:82-94.
43. Joo I, Lee JM, Lee DH, Jeon JH, Han JK. Retrospective validation of a new diagnostic criterion for hepatocellular carcinoma on gadoxetic acid-enhanced MRI: can hypointensity on the hepatobiliary phase be used as an alternative to washout with the aid of ancillary features? Eur Radiol 2019;29:1724-1732.

44. Maruyama H, Sekimoto T, Yokosuka O. Role of contrast-enhanced ultrasonography with Sonazoid for hepatocellular carcinoma: evidence from a 10-year experience. J Gastroenterol 2016;51:421-433.

45. Hatanaka K, Kudo M, Minami Y, Maekawa K. Sonazoid-enhanced ultrasonography for diagnosis of hepatic malignancies: comparison with contrast-enhanced CT. Oncology 2008;75 Suppl 1:42-47.

46. Liu W, Qin J, Guo R, Xie S, Jiang H, Wang X, et al. Accuracy of the diagnostic evaluation of hepatocellular carcinoma with LI-RADS. Acta Radiol 2018:59:140-146.

47. Kim YY, An C, Kim S, Kim MJ. Diagnostic accuracy of prospective application of the Liver Imaging Reporting and Data System (LI-RADS) in gadoxetate-enhanced MRI. Eur Radiol 2018;28:2038-2046.

48. Abd Alkhalik Basha M, Abd El Aziz El Sammak D, El Sammak AA. Diagnostic efficacy of the Liver Imaging-Reporting and Data System (LI-RADS) with CT imaging in categorising small nodules (10-20 $\mathrm{mm}$ ) detected in the cirrhotic liver at screening ultrasound. Clin Radiol 2017;72:901.e1-901.e11.

49. Sofue K, Burke LMB, Nilmini V, Alagiyawanna M, Muir AJ, Choudhury $K R$, et al. Liver imaging reporting and data system category 4 observations in MRI: risk factors predicting upgrade to category 5. J Magn Reson Imaging 2017;46:783-792.

50. Tanabe M, Kanki A, Wolfson T, Costa EA, Mamidipalli A, Ferreira $M P$, et al. Imaging outcomes of liver imaging reporting and data system version 2014 category 2, 3, and 4 observations detected at CT and MR imaging. Radiology 2016;281:129-139.

51. Burke LM, Sofue K, Alagiyawanna M, Nilmini V, Muir AJ, Choudhury $K R$, et al. Natural history of liver imaging reporting and data system category 4 nodules in MRI. Abdom Radiol (NY) 2016;41:1758-1766.

52. Bruix J, Sherman M; Practice Guidelines Committee, American Association for the Study of Liver Diseases. Management of hepatocellular carcinoma. Hepatology 2005;42:1208-1236.

53. Zhang J, Yu Y, Li Y, Wei L. Diagnostic value of contrast-enhanced ultrasound in hepatocellular carcinoma: a meta-analysis with evidence from 1998 to 2016. Oncotarget 2017;8:75418-75426.

54. Mitchell DG, Bruix J, Sherman M, Sirlin CB. LI-RADS (Liver Imaging Reporting and Data System): summary, discussion, and consensus of the LI-RADS Management Working Group and future directions. Hepatology 2015;61:1056-1065.

55. Jang KM, Kim SH, Kim YK, Choi D. Imaging features of subcentimeter hypointense nodules on gadoxetic acid-enhanced hepatobiliary phase MR imaging that progress to hypervascular hepatocellular carcinoma in patients with chronic liver disease. Acta Radio 2015;56:526-535. 
Tae-Hyung Kim, et al. Guideline for HCC diagnosis, 2018 update

56. Song KD, Kim SH, Lim HK, Jung SH, Sohn I, Kim HS. Subcentimeter hypervascular nodule with typical imaging findings of hepatocellular carcinoma in patients with history of hepatocellular carcinoma: natural course on serial gadoxetic acid-enhanced MRI and diffusionweighted imaging. Eur Radiol 2015;25:2789-2796.

57. Wang JH, Changchien CS, Hu TH, Lee CM, Kee KM, Lin CY, et al.
The efficacy of treatment schedules according to Barcelona Clinic Liver Cancer staging for hepatocellular carcinoma - Survival analysis of 3892 patients. Eur J Cancer 2008;44:1000-1006.

58. Joo I, Lee JM. Recent advances in the imaging diagnosis of hepatocellular carcinoma: value of gadoxetic acid-enhanced MRI. Liver Cancer 2016;5:67-87. 